\title{
PR-26. SIMULATION RESULTS-SOURCE FOR THE IDENTIFICATION OF BIOLOGICAL ACTIVE COMPOUNDS: SYNTHESIS, ANTIMICROBIAL EVALUATION AND SARS OF THREE IN ONE HETEROCYCLIC MOTIFS
}

\author{
G. Sravya $^{1}$, G. V. Zyryanov ${ }^{1,2}$ \\ ${ }^{1}$ Ural Federal University of the first President of Russia B. N. Yeltsin, \\ Mira St., 19, Yekaterinburg, 620002, Russia \\ ${ }^{2}$ I. Ya. Postovsky Institute of Organic Synthesis UB RAS, \\ S. Kovalevskoy/Akademicheskaya St., 20/22, Yekaterinburg, 620990, Russia \\ E-mail: sravyasvu@gmail.com
}

Abstract. For studies on drugs and biomimetic systems electronic parameters becoming more efficient and further powerful tools. In this perspective, the present work synthesis, antimicrobial screening and simulation studies of three-in-one heterocyclic derivatives have been taken up. This successful work demonstrated that, the compounds $\mathbf{5 d}$ and $\mathbf{7 d}$ have the higher activity and it was confirmed by the help of frontier molecular orbital particularly LUMO because of LUMO as an electron withdrawing nature. In fact, the biological activity was justified by simulation studies.

Many bioassay compounds in that benzimidazoles are resourceful lead structures in molecular drug design of antibacterials, antivirals, fungicides, and antimutagens. The benzimidazole based molecules have been reported to have potential anticancer activities against different types of cancers. In that, Bendamustine and Veliparib are drugs approved for the cancers treatment, also, different substitutions on benzimidazole and its derivatives have been used to analyze their therapeutic potential. Moreover, the oxazole ring is gifted with various activities such as hypoglycemic, analgesic, anti-inflammatory and antibacterial. The oxazole nucleus is also ubiquitous in natural products such as pyrronazol, ulapualides, diazonamides, and rhizopodin. In addition, oxazole derivatives have exhibited a high potential as efficient luminophores for liquid and fluorescent probes and markers in biological or supramolecular systems. Oxazole ring is privileged scaffolds for the generation of target compounds for drug discovery. Motivated by the above interpretation and our continued attention in the progress of above heterocycles in one moiety to build up bioactive heteroaromatic compounds, the present, work to synthesize a new class of benzimidazole containing pyrazolyl oxazoles is described as potential antimicrobials and correlated with their electronic parameters.

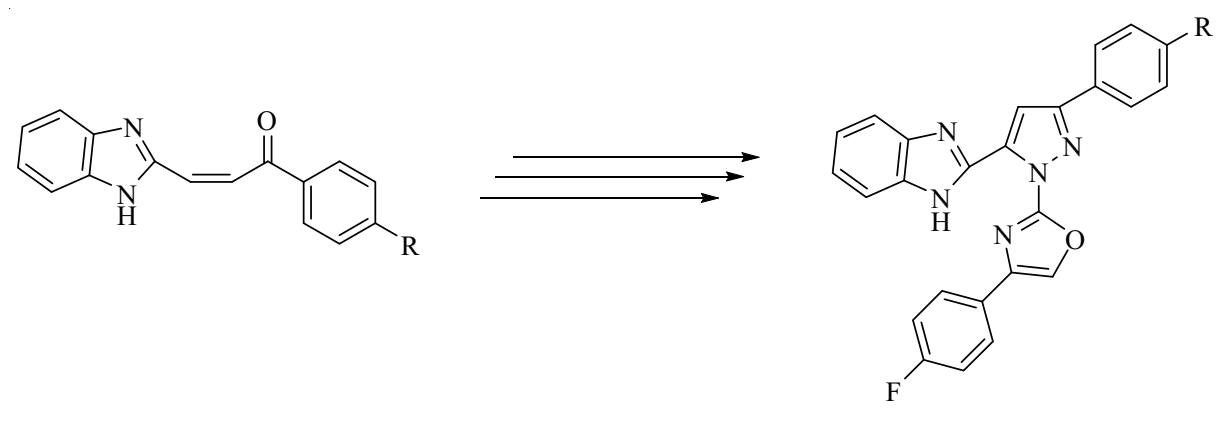

$\mathrm{R}=\mathrm{H}, \mathrm{Me}$, OMe, $\mathrm{NO}_{2}, \mathrm{~F}, \mathrm{Cl}, \mathrm{Br}$

Synthesis of benzimidazolyl pyrazoles and benzimidazolyl pyrazolyl oxazoles 